\title{
Comparsion of traditional and modern dressing methods on children with deep burn injury after autologous transplant
}

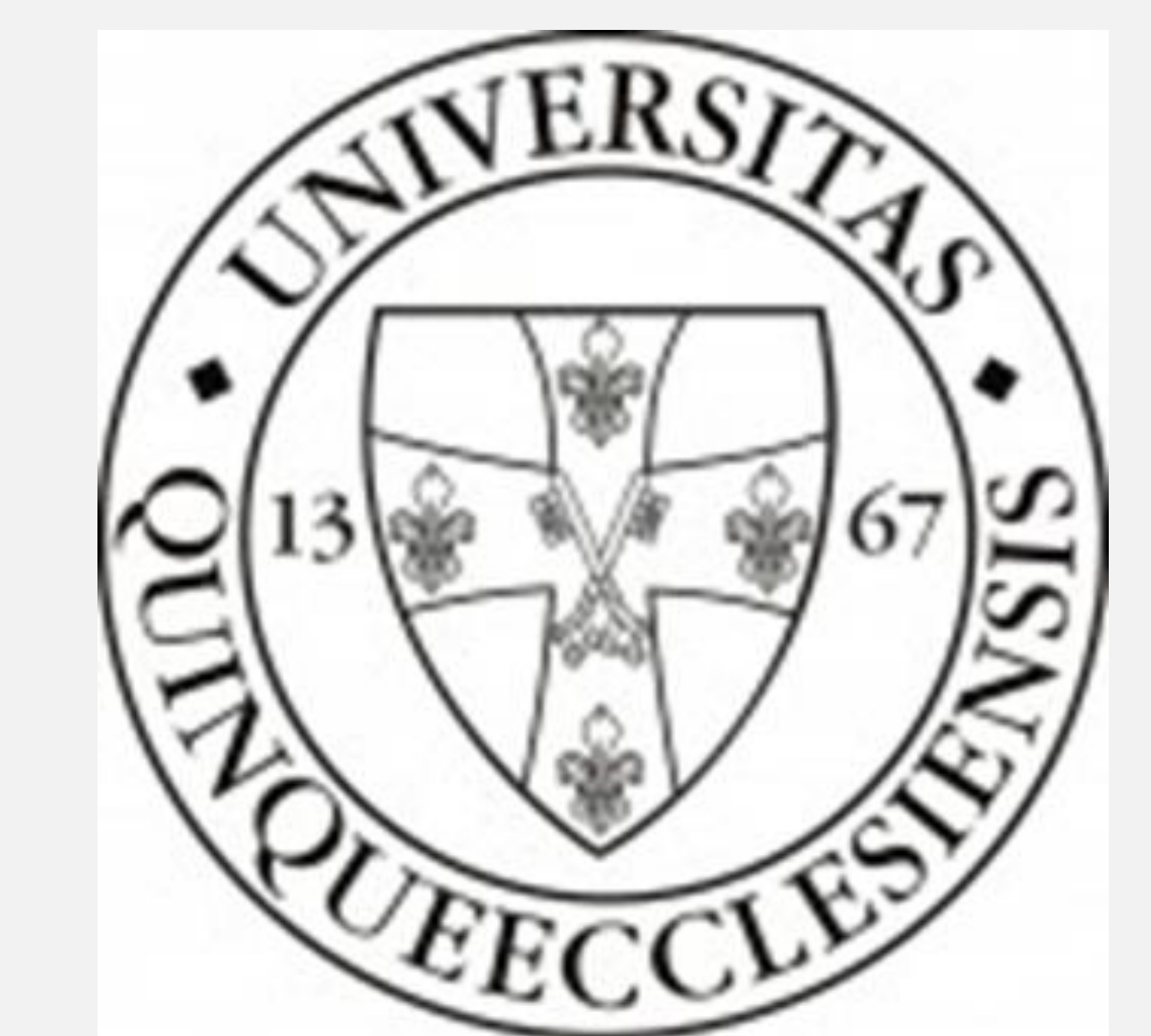

\section{Gergo Jozsa, Zsolt Juhasz}

Division of Pediatric Surgery, Traumatology, Urology and Pediatric Otolaryngology, Department of Pediatrics, Medical Centre, University of Pécs, Hungary

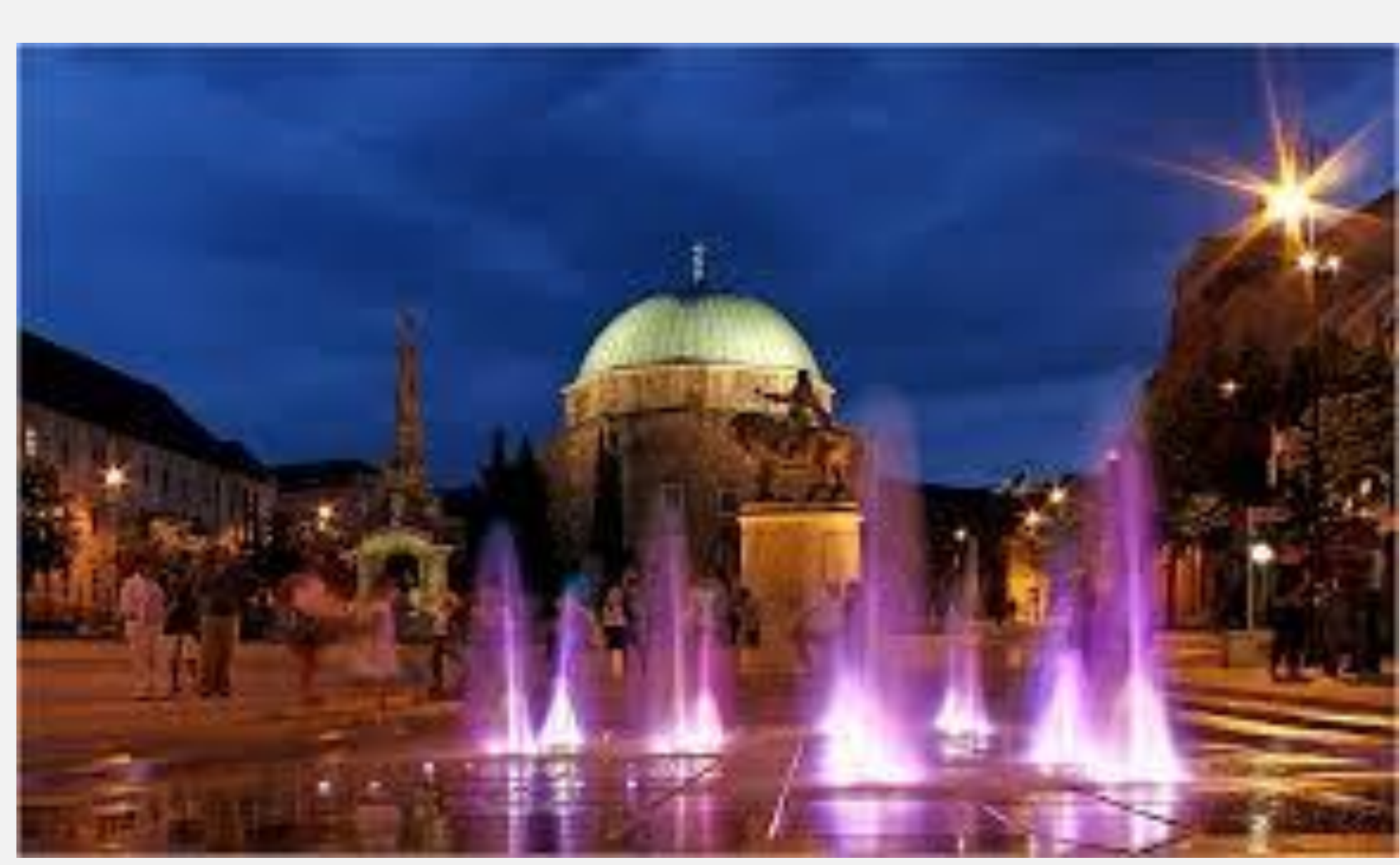

Introduction: Burns is a common type of traumatic injury in childhood. Nowadays, several treatment options are available to treat after the autolog skin grafting because of the deep burn injury.

Aims: Comparison of the postoperative treatment of the patients who were transplanted because of deep (II/2, III degree) burn injuries.

Methods: Retrospective study has been carried out between January 1, 2015 and December 1, 2017. A total of 16 transplantation was performed because of deep burn injury. This study was a single-arm, retrospective case series study. The institutional review board provided approval prior to participant recruitment. All study procedures were conducted at Pediatric center in Pécs in Hungary. Two groups were developed for the retrospective study. In Group 1 Grassolind mesh with Betadine solution (Figure 1.), while in Group 2 Aquacel Ag foam dressing with Curiosa gel (Figure 2.) were applied to cover the transplanted skin.

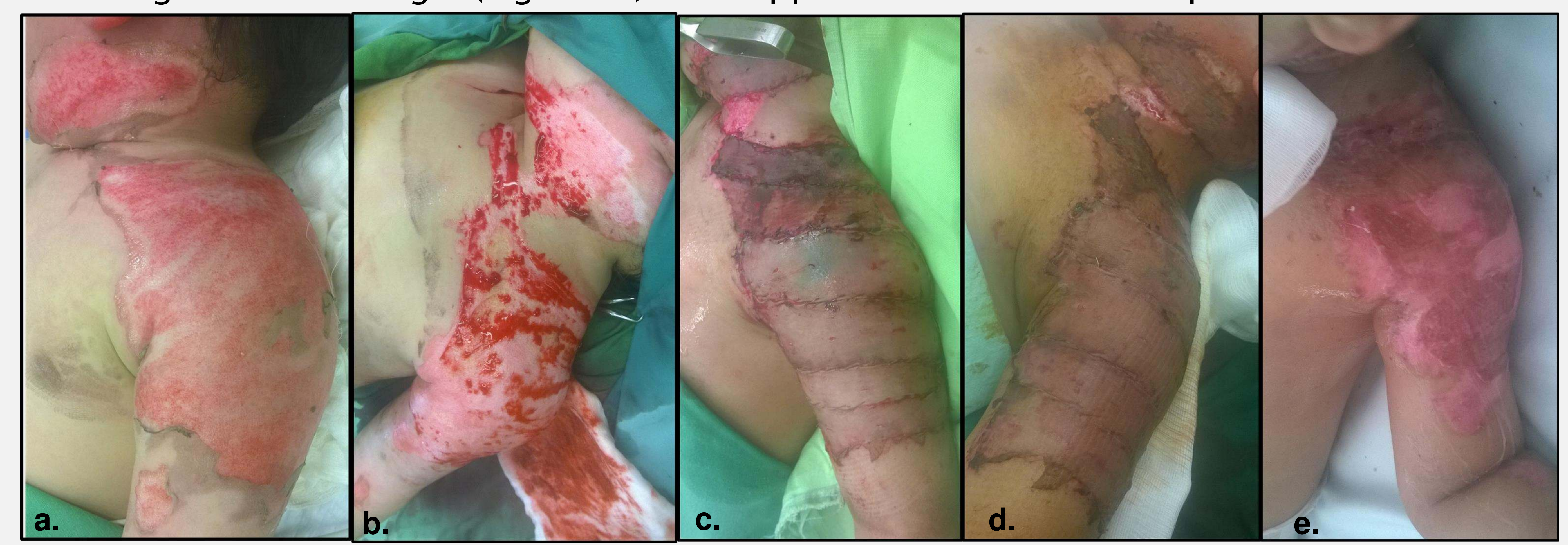

Figure 1.: Treatment of Group I patient: Mixed second degree burn injury of the left upper limb and neck before, (a.) and after the necrectomy (b.) 3days after the skin grafting (c.). 7 days after the transplantation (d.), One month after the injurythe cosmetic result is good (e.).

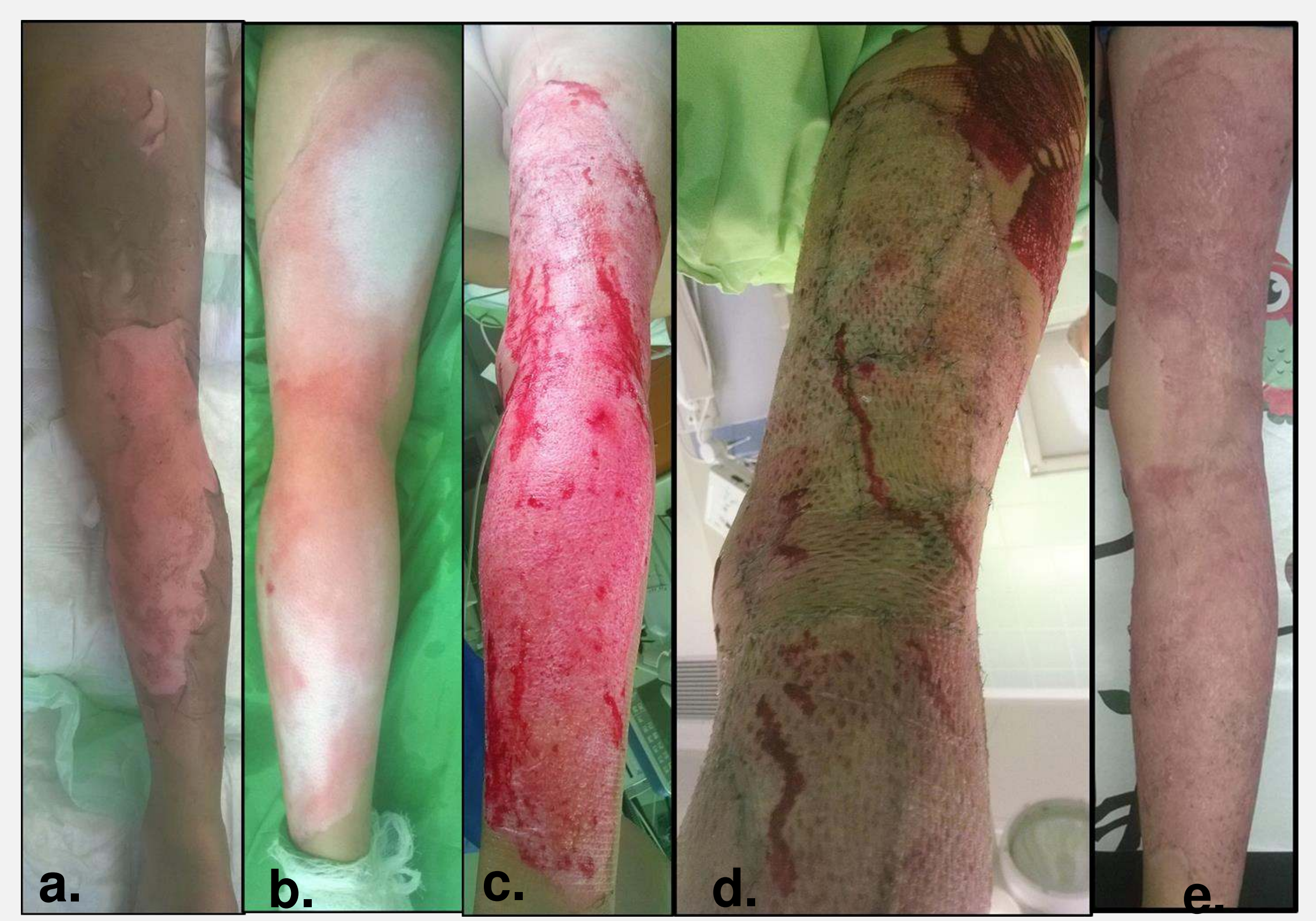

Figure 2.:Treatment of Group I patient: Third degree burn injury of the dorsal egion of the right lower limb (a.) before (b.) and after the necrectomy (c.) 2 cosmetic result is good (e.).

Results: In Group 1 seven children, while in Group 2 nine children were involved in the clinical study. The average age were under 5 years in both groups (Figure 3.). The causes of the injury were scalding with hot liquids in all cases in Group 1(Figure 7.). The extent of the burn in three cases was 5-10\%. The children spent average 21.9 days in hospital (Figure 6.), meanwhile average six anesthesia were performed to change the bandage (Figure 5.). The removal of the bandage usually happened on the 13th day (Figure 4.). Four children were injured because of scalding, while 5 children suffered from contact burns in Group 2 (Figure 7.). The extent of the injury in four cases was less than 5\%, in two cases was 5-10\%, and in three cases was more than 10\%. The children spent average 13.8 days in the hospital (Figure 6.), meanwhile average three anesthesia were performed on them (Figure 5.). The removal of the bandage usually happened on the 10th day (Figure 4.).

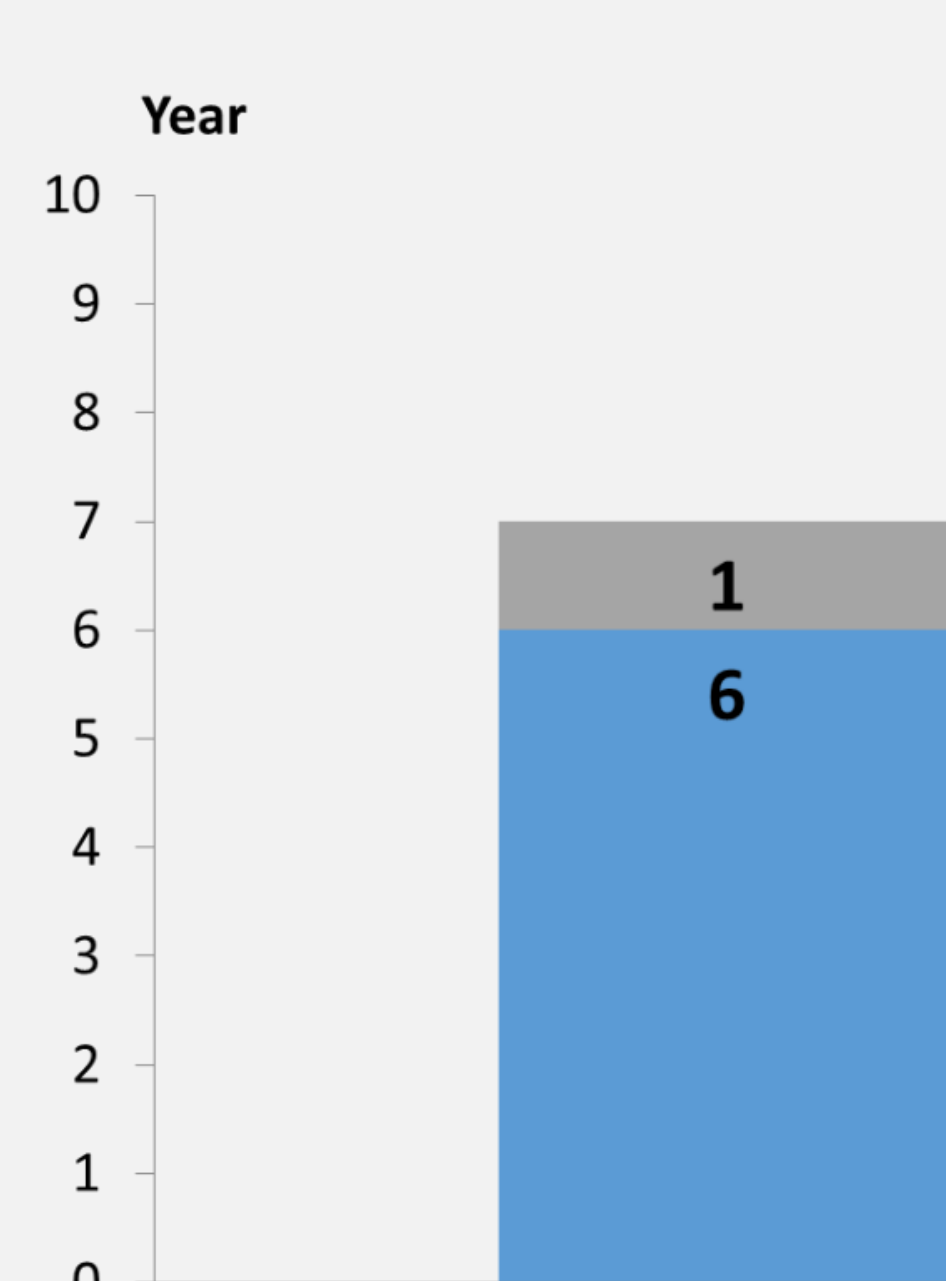

Group I

Figure 3.: Age distribution of the study population.

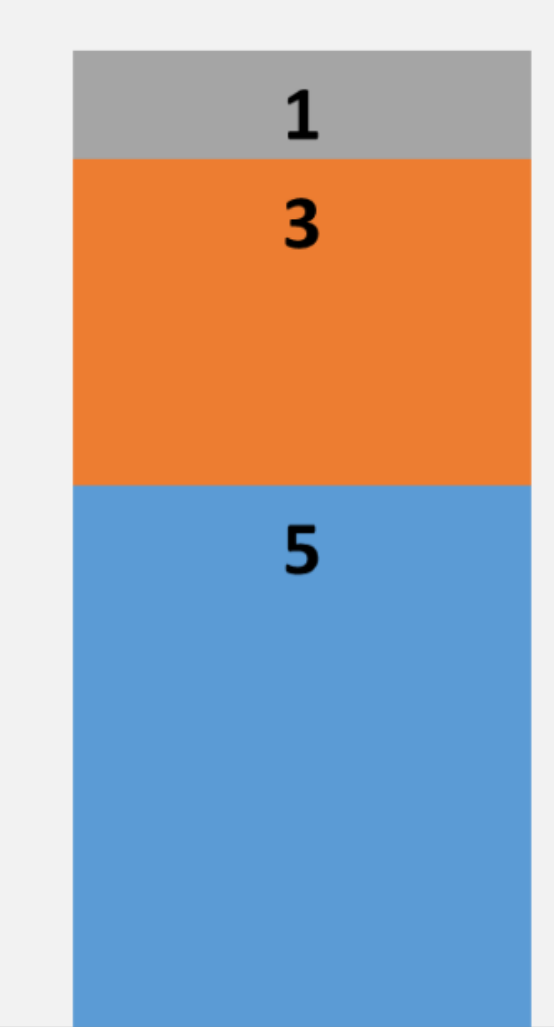

Group II

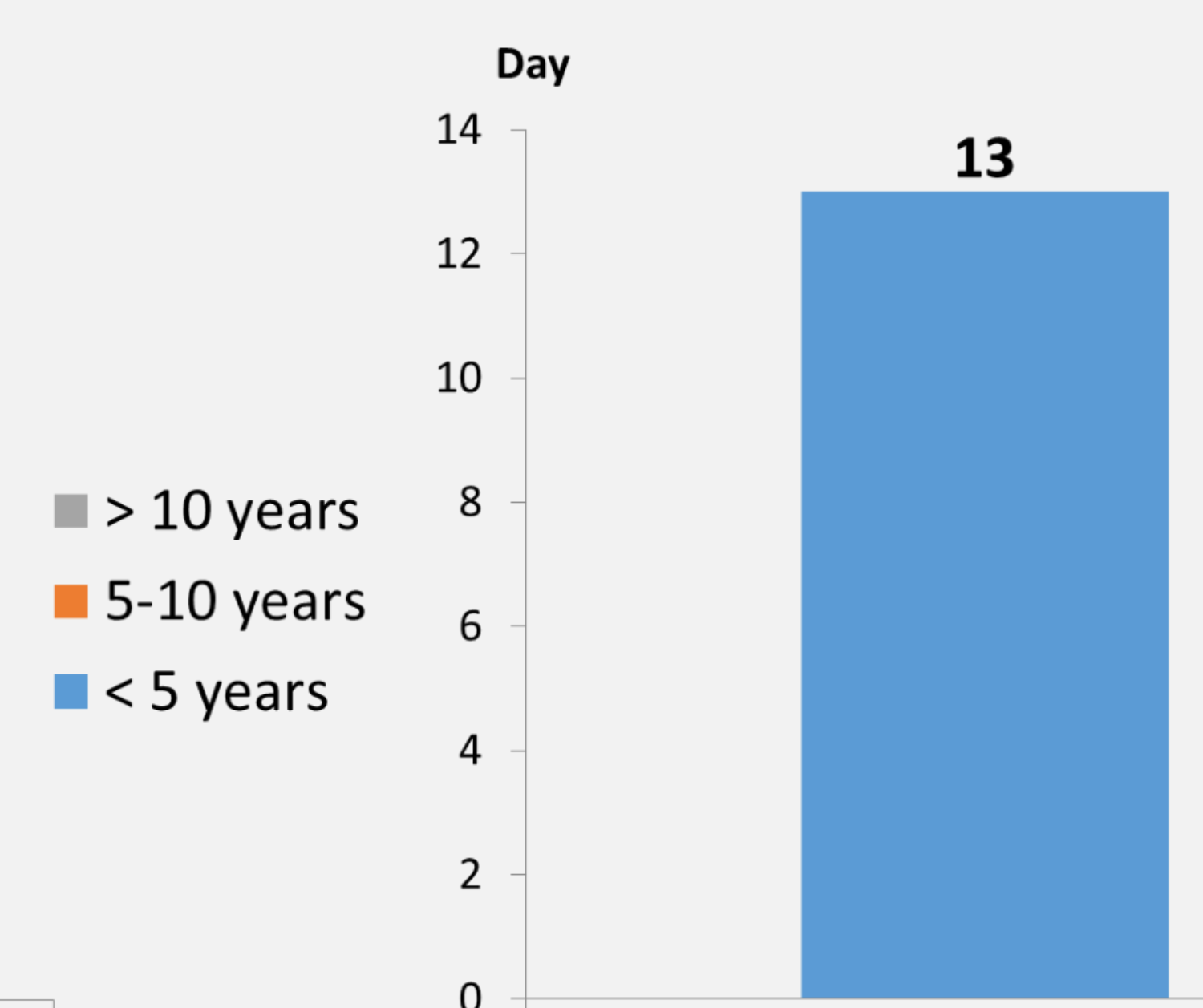

Group I

Figure 4.: Avarege time of the healing.
Number $\left(N^{\circ}\right) \quad$ Avarege number of the anesthesia
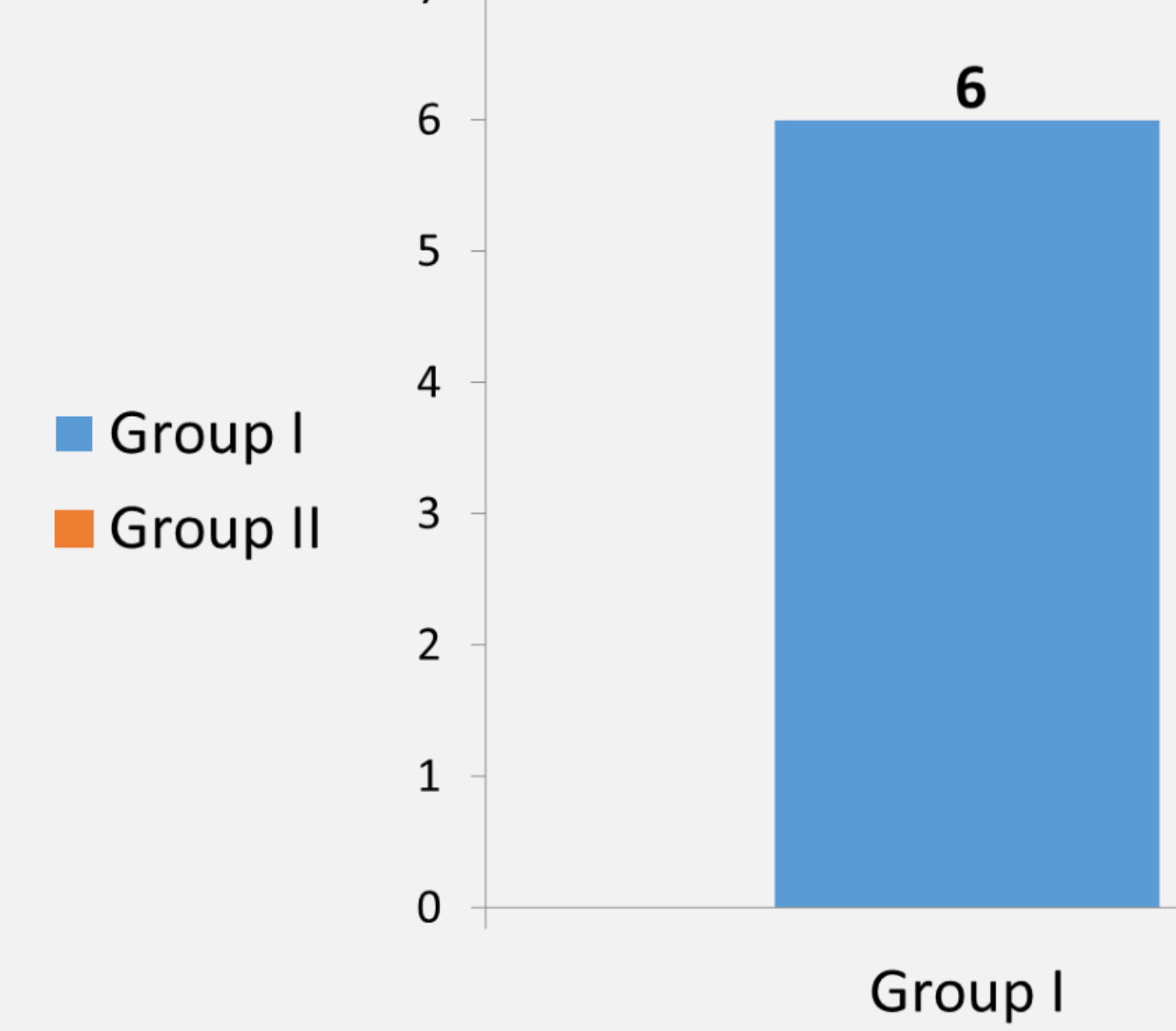

Group I

Figure 5.: Avarege number of the anesthesia.

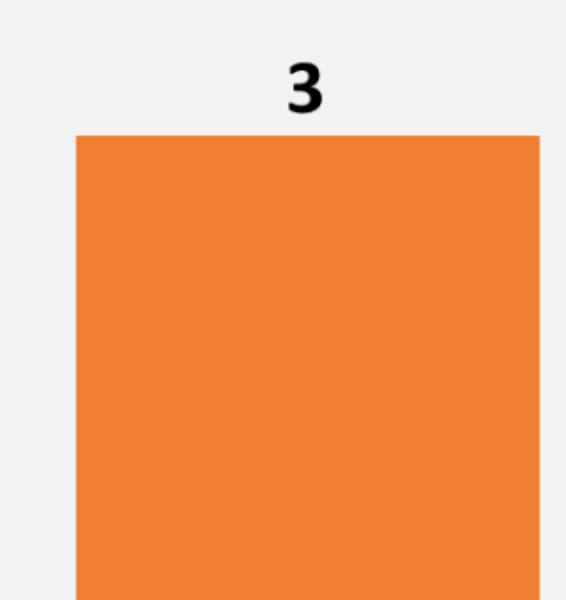

Group II \section{(1) \\ .}

Causes of the injury - Group I Causes of the injury - Group II

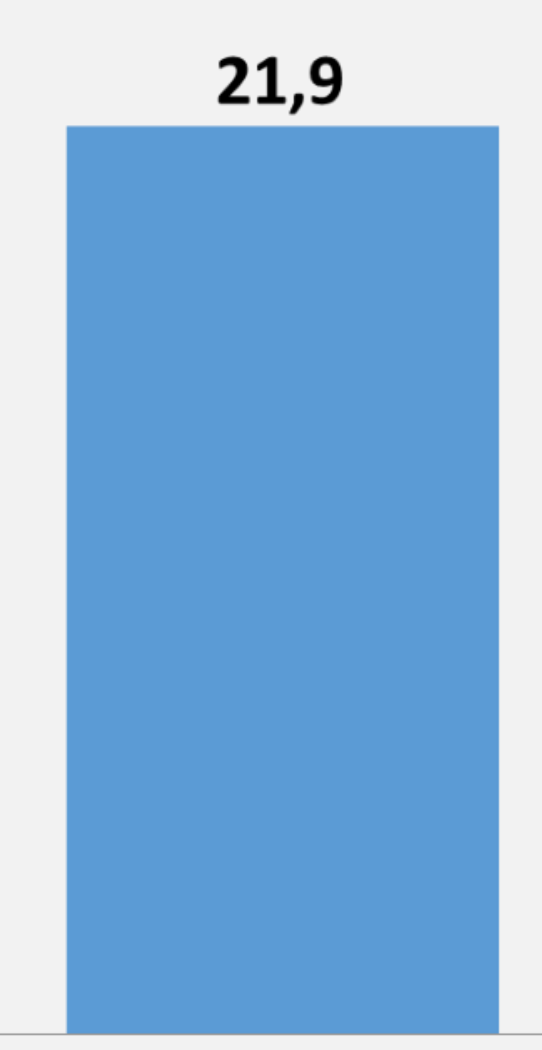

Group I

Figure 6.: Hospitalisation time.
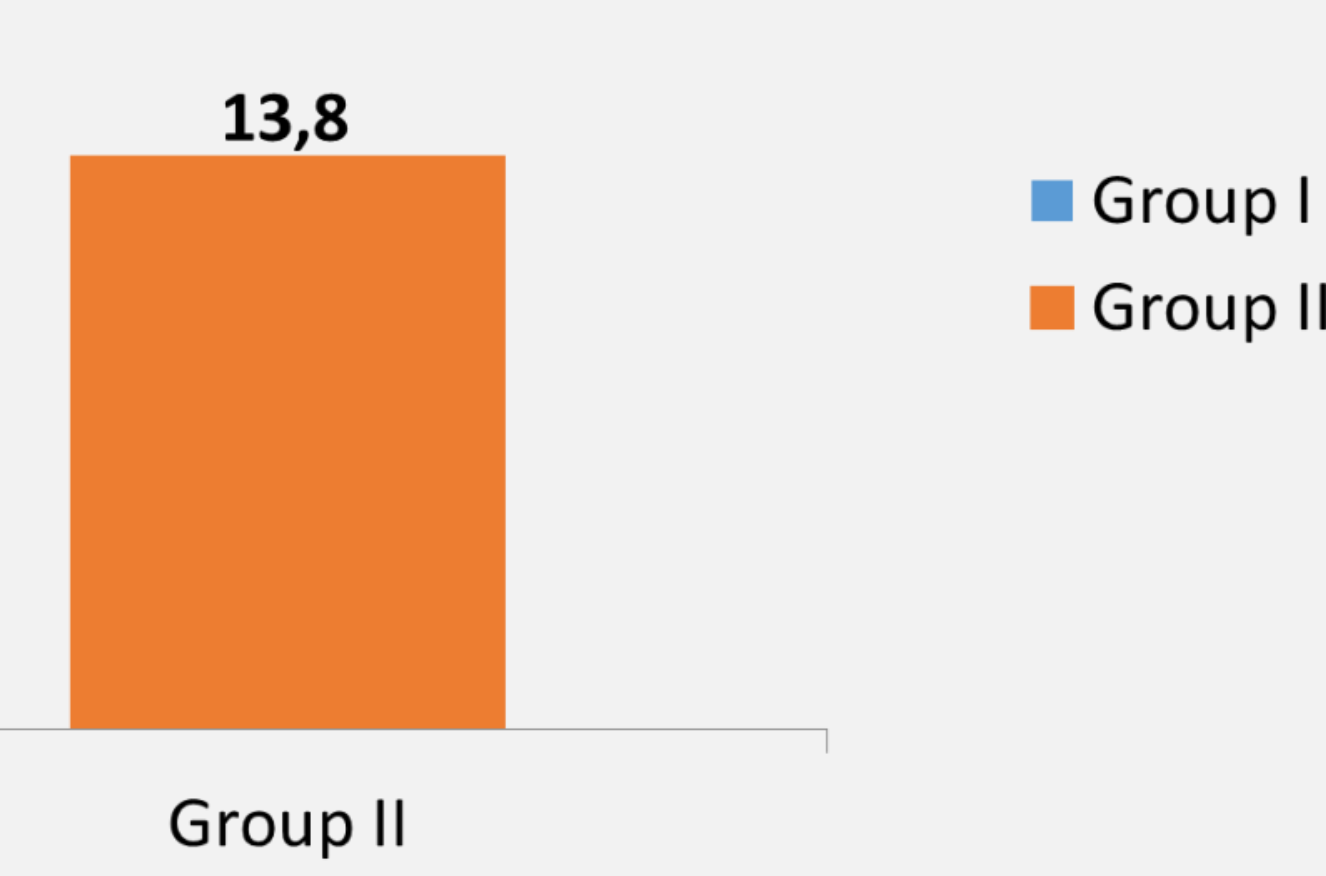

Group I

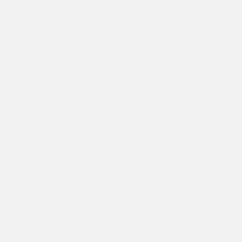
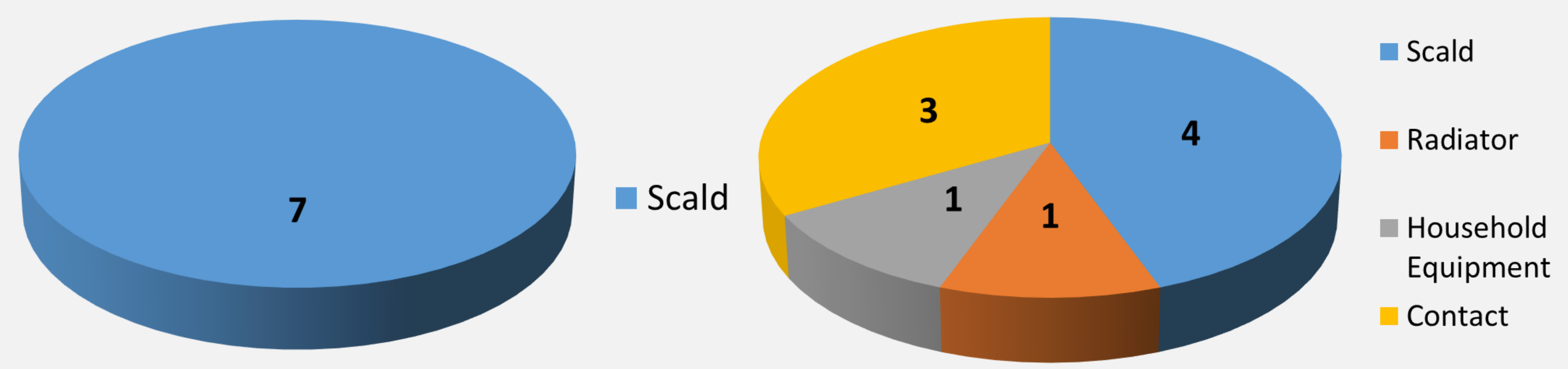

Figure 7: Causes of injury in the study population.

Conclusion: In case of a II/2, III degree deep burn injury, after the required transplantation and the use of the Aquacel Ag foam dressing and Curiosa gel resulted in significant decrease of the number of performed anaesthesias and the days spent in the hospital. In Group 2 , where the intelligent bandage was used, the final removal happened 3 days earlier.

Gergo Jozsa, MD - Unit of Pediatric Surgery, Traumatology and Urology, Department of Pediatrics, Medical Centre, University of Pécs, Hungary E-mail: dr.jozsa.gergo@gmail.com 